\title{
RESEARCH
}

Open Access

\section{Correlation of cortical lesions of multiple sclerosis at double inversion recovery with cognition screening scores}

Sally Mohamed Shaaban ${ }^{1 *} \mathbb{D}$, Azza Elmongui Elmongui', Ahmed Abdel Khalek Abdel Razek² and Tamer Mohamed Belal ${ }^{1}$

\begin{abstract}
Background: Multiple sclerosis is a chronic inflammatory disease affecting both white and gray matters of the central nervous system. It has been approved that the degree of gray matter involvement is closely associated with the degree of physical disability and the extent of cognitive impairment. Thus, it is necessary to incorporate widely available simple methods for neurocognitive evaluation and gray matter detection in the periodic assessment of MS patients that will influence treatment decisions.
\end{abstract}

Objectives: To assess the correlation of cortical lesions of multiple sclerosis (MS) at double inversion recovery (DIR) with cognition screening scores

Methods: This study was conducted on 30 patients with MS with an average age of $31.3 \pm 13.6$ years. All of them underwent MRI and clinical assessment with the calculation of Expanded Disability Status Scale (EDSS), Montreal Cognitive Assessment (MoCA), and Symbol Digit Modality Test (SDMT) scores. The image analysis was performed by 2 reviewers for cortical lesion number, shape, and subtypes, and total lesion load.

Results: Both MoCA and SDMT scales had a significant inverse correlation with cortical lesions number ( $r=-0.68,-$ $0.72)$ respectively and total lesion load $(r=-0.53,-0.65)$ respectively. Besides, there was a significant inverse correlation between the MoCA test, varied cortical subtypes: leukocortical, juxtacortical, and intracortical subtypes ( $r$ $=-0.63,-0.56,-0.52)$ respectively, and different cortical lesion shapes: oval, wedge, and curvilinear shaped ( $r=-$ $0.62,-0.69,-0.49)$ respectively. As well, the SDMT scale showed a significant inverse correlation with varied cortical subtypes: intracortical, leukocortical, and juxtacortical subtypes $(r=-0.63,-0.61,-0.57)$ respectively, and different cortical lesion shapes: oval, curvilinear, and wedge shaped $(r=-0.61,-0.59,-0.46)$ respectively. Interestingly, there was an excellent inter-observer correlation of cortical lesion number $(r=0.96)$, total lesion load $(r$ $=0.95)$, subtypes of cortical lesion $(r=0.94)$, and cortical lesion shapes $(r=0.77)$.

Conclusion: We concluded that DIR can detect cortical lesions of MS, and MRI findings were well-correlated with cognitive dysfunction in these patients.

Keywords: Multiple sclerosis, Cortical lesions, Cognitive impairment, Double inversion recovery

\footnotetext{
* Correspondence: sallyshaaban@mans.edu.eg

${ }^{1}$ Department of Neurology, Mansoura Faculty of Medicine, Mansoura, Egypt

Full list of author information is available at the end of the article
}

\section{Springer Open}

(c) The Author(s). 2021 Open Access This article is licensed under a Creative Commons Attribution 4.0 International License, which permits use, sharing, adaptation, distribution and reproduction in any medium or format, as long as you give appropriate credit to the original author(s) and the source, provide a link to the Creative Commons licence, and indicate if changes were made. The images or other third party material in this article are included in the article's Creative Commons licence, unless indicated otherwise in a credit line to the material. If material is not included in the article's Creative Commons licence and your intended use is not permitted by statutory regulation or exceeds the permitted use, you will need to obtain permission directly from the copyright holder. To view a copy of this licence, visit http://creativecommons.org/licenses/by/4.0/. 


\section{Introduction}

Multiple sclerosis (MS) is a chronic demyelinating and neurodegenerative disease of the central nervous system which strikes young adults and imposes major burdens on their lives through physical disability and cognitive deficit $[1,2]$. Cortical lesions in MS are classified pathologically according to their location into subpial, purely intracortical, leukocortical, and pancortical lesions [3]. Besides, it has been proven that these cortical lesions are specific for MS [4, 5]. Therefore, the most recent revisions of McDonald's criteria, issued in 2017, have incorporated these lesions into MS diagnostic criteria to demonstrate dissemination in space [6]. The gray matter pathology plays a major role in cognitive impairment [7]. Cognitive impairment reveals a significant correlation with physical disability accumulation [8]. Inclusion of neurocognitive evaluation and gray matter detection in the periodic assessment of MS patients is mandatory to detect patients at increased risk of secondary progression that will influence treatment decisions regarding diseasemodifying drugs and rehabilitation therapy [9]. Neurocognitive screening tests such as Montreal Cognitive Assessment (MoCA) and Symbol Digit Modality Test (SDMT) are easy to assess cognitive status in MS patients $[10,11]$. Moreover, double inversion recovery (DIR) is a common MRI pulse sequence that is proven to be superior to FLAIR sequence in detecting MS lesions in different locations especially cortical, periventricular, and infratentorial regions [12]. It is designed to suppress the signal of surrounding white matter and cerebrospinal fluid (CSF) allowing the detection of gray matter lesions especially cortical lesions $[13,14]$.

\section{Methods}

This is a cross-sectional study that was conducted upon MS patients admitted at our neurology department from May 2017 to June 2018. The study was conducted upon 34 MS patients meeting revised 2017 McDonald's criteria [6]. This study included only 30 MS patients while 4 patients were excluded from older patients who were over 50 years $(n=2)$ and illiterate patients $(n=2)$. The research assessment of patients was performed by one neurologist (SS) with 4 years' experience in clinical practice. Physical disability was assessed by EDSS [15]. While the Brief International Cognitive Assessment in MS (BICAMS) is the established approach for screening for cognitive impairment in MS, the authors of our study preferred to use the highly sensitive cognitive screening tools (MoCA and SDMT), as they are more broadly applicable in the outpatient clinic, briefer to administer, and easier to score than BICAMS with a single value cut point to determine impairment. MoCA is a short test of 30 points that evaluates 8 cognitive domains, including visuospatial abilities, executive functions, naming, attention, language, abstraction, short-term memory, and orientation. The score is a total of the earned points with the cutoff score of less than 26 and 19 indicate mild cognitive impairment (MCI) and dementia, respectively; people whose education is 12 years or less obtained an extra point $[16,17]$. SDMT is used to assess information processing speed; it consists of a sheet of paper with, at the top, a sequence of nine symbols and nine corresponding numbers. The task sequence consists of a series of digits, each with a blank space underneath. The subject should rapidly match numbers to abstract symbols using a key. The total duration should not exceed $90 \mathrm{~s}$. The raw score is the total of correctly answered items with a cutoff $Z$-score of less than 7 [18]. The MR imaging examinations were performed on a 1.5-Tesla machine (Achieva; Philips Medical Systems, Best, Netherlands). All patients were examined in the supine position using the head coil. Routine images ( $\mathrm{T} 1$, T2, FLAIR) and DIR were done to all patient: repetition time (TR) $9583 \mathrm{~s}$, echo time (TE) $25 \mathrm{~s}$, inversion time (TI) $3400 \mathrm{~s}$, IR delay $325 \mathrm{~s}$, echo train length (ETL) 17, 50 contiguous axial slices, thickness $3 \mathrm{~mm}$, matrix size $240 \times 142 \mathrm{~mm}^{2}$, the field of view (FOV) $230 \times 184 \mathrm{~mm}^{2}$. Image analysis was performed by 2 observers: one neurologist (SS) (observer 1) with 4 years' experience and one neuro-radiologist (AA) (observer 2) with 20 years' experience who were blinded to patient identity. On DIR images, particular attention was devoted to identifying artifacts. Cortical lesions were identified on DIR using the corresponding FLAIR images as a reference. First, cortical lesions were defined as those lesions involving the cortex with or without extension to the underlying subcortical WM, and their total number [19] was counted. Then, these lesions were categorized according to their shapes into an oval, curvilinear, and wedge shaped [20] and their locations into three subtypes including purely intracortical (IC) in which there was no obvious evidence of white matter involvement, leukocortical (LC) where most of the lesion volume was intracortical with clear evidence of some extension into the white matter, and juxtacortical (JC) lesion that was mainly subcortical abutting the cortical gray matter [21]. After that, a quantitative assessment of the total MS lesion load in both white and gray matters was done by manual segmentation of lesions [22]. Manual segmentation was done lesion by lesion and slice by slice on DIR sequence on axial slices using a freehand drawing region of interest (ROI) method. The application used for ROI drawing enables users to scale the image size and adjust brightness for viewing. After edge detection and contouring 
of each lesion, this segmentation was saved. The total surface area (SA) of all lesions was calculated; then, the total lesion load was evaluated (by multiplying total SA $\times 3 \mathrm{~mm}$ representing slice thickness and interslice gaps).

The collected data were coded, processed, and analyzed using the IBM SPSS statistics (Statistical Package for Social Sciences) software version 22.0, IBM Corp., Armonk, USA, 2013. Qualitative data were presented as numbers and percentages. A comparison among groups was done by the Chi-square test. Quantitative data were tested for normality by the Kolmogorov-Smirnov test. Normally distributed data were presented as mean \pm SD . The Student $t$-test was used to compare two groups. Cohen's kappa ( $\mathrm{\kappa}$ ) was run to determine if there was an agreement between the two cognitive screening tests (MoCA and SDMT) on whether $30 \mathrm{MS}$ patients were exhibiting normal or impaired cognitive function. Values less than 0.40 are indicative of a fair agreement, values between 0.41 and 0.60 indicate a moderate agreement, values between 0.61 and 0.80 indicate a good agreement, and values greater than 0.80 indicate a very good agreement to examine the degree of inter-observer correlation on MRI data; correlation analysis was used to produce inter-observer correlations. Values less than 0.5 are indicative of poor correlation, values between 0.5 and 0.75 indicate moderate correlation, values between 0.75 and 0.9 indicate good correlation, and values greater than 0.90 indicate an excellent correlation. MRI data collected by observer 2 was used in the correlational analysis. The Welch ANOVA test was used to compare the mean CL number with different cognitive status assessed by MoCA. A multivariate backward linear regression analysis was used to detect independent predictors of MoCA and SDMT. A $p$-value of less than 0.05 was considered statistically significant.

\section{Results}

Patients in this study were relapsing-remitting (RR) MS patients (23) and secondary progressive (SP) MS patients (7). The mean and standard deviation (SD) of the disease duration was $5.3 \pm 3.2$. The mean and SD of EDSS was $3.1 \pm 1.5$. As regards cognitive screening tests, the mean and SD of scores of the MoCA and the SDMT were 23.5 \pm 3.8 and $8.2 \pm 2.6$, respectively (Table 1 ). Moreover, there was a moderate agreement between the MoCA and the SDMT on cognitive screening for MS patients, $k$ $=0.553$ (95\% CI, 0.294 to 0.812), $p=0.001$. Thus, both tests have agreed on $10 \mathrm{MS}$ patients having intact cognition and 13 MS patients exhibiting cognitive impairment. However, 7 MS patients were diagnosed cognitively impaired using MoCA while they normally scored by SDMT. Therefore, the cases diagnosed cognitively intact by MoCA test also were diagnosed the same
Table 1 Clinical characteristics of the studied patients

\begin{tabular}{lll}
\hline & Number & $\%$ \\
\hline Ambulation & & \\
Ambulant $\left(E^{2} S^{\text {a }}=0-4.5\right)$ & 23 & 76.7 \\
Ambulatory problems (EDSS $\geq 5)$ & 7 & 23.3 \\
MoCA ${ }^{\text {b }}$ & & \\
$\quad$ Intact cognition & 3 & 10 \\
Impaired cognition & 27 & 90 \\
SDMT ${ }^{\text {c }}$ & & \\
Intact cognition & 17 & 56.7 \\
Impaired cognition & 13 & 43.3 \\
\hline
\end{tabular}

${ }^{a}$ Expanded disability status scale

${ }^{\mathrm{b}}$ Montreal Cognitive Assessment

'Symbol Digit Modality Test

by SDMT. Among the 20 MS patients with impaired cognitive function, there were 4 of them diagnosed with dementia by MoCA. However, on using the SDMT, one of them had a score of 5 while the other three patients had a score of 6 . Besides, the remaining sixteen patients had mild cognitive impairment by MoCA; however, on using the SDMT, one of them had a score of 5, eight patients obtained a score of 6 , while the remaining seven patients had normal scores. Regarding DIR findings, the total number of cortical lesions assessed by both observers was (544, 633 respectively) associated with an excellent inter-observer correlation $(r=0.96 ; p=0.001)$. The most common type of cortical lesions noted by both observers presented in Fig. 1 is JC followed by IC then $\mathrm{LC}$ with an excellent inter-observer correlation $(r=0.94$; $p=0.001)$. Moreover, the most common shape of cortical lesions presented in Fig. 2 is oval followed by wedge then curvilinear shaped with good inter-observer correlation ( $r=0.77 ; p=0.001$ ) (Table 2). The mean of total lesion load detected by both observers presented in Fig. 3 is 10.82 and $10.93 \mathrm{ml}$ respectively with an excellent inter-observer correlation $(r=0.95 ; p=0.001)$. Only $20 \%$ of our patients had a high total lesion load as assessed by two observers. Regarding the correlation of cognitive tests with other patient variables, MoCA showed a positively significant correlation with the disease duration and EDSS. Also, there was an inversely significant correlation detected between the MoCA test and MRI variables involving the number of cortical lesions, different cortical lesion shapes, various subtypes of cortical lesions, and the assessed total lesion load (Table 3). SPMS patients were cognitively impaired (mean $\pm \mathrm{SD}=19 \pm$ 4.9) more severe than RRMS patients (mean $\pm \mathrm{SD}=24.9$ \pm 1.8 ) with significant value $(p=0.019)$. As regard to different cognitive status assessed by MoCA, there was no statistically significant difference in mean CL numbers between dementia (mean $\pm \mathrm{SD}, 44.8 \pm 16.3$ ) versus MCI (mean \pm SD, 23.6 \pm 17.4) patients. However, the mean 

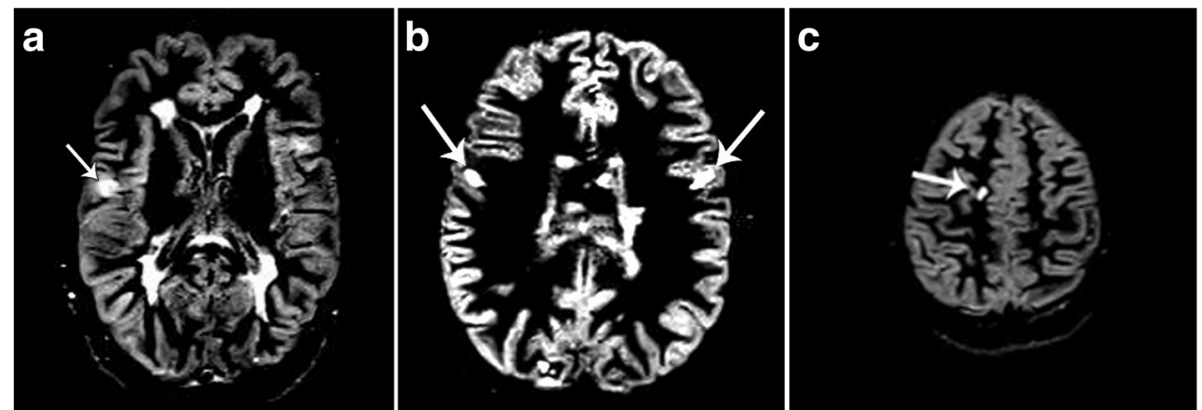

Fig. 1 Axial DIR images showing cortical lesions location: a Intracortical lesion in the right temporal region. b Two leukocortical lesions in the right and left frontoparietal regions. c Juxtacortical lesions in the right prefrontal regions

CL number was statistically significantly higher for dementia as well as $\mathrm{MCI}$ versus cognitively normal (mean \pm SD, $7.7 \pm 5.5$ ) patients (Welch $F=13.3, p=0.004$ ) (Fig. 4). As well, SDMT showed a significant inverse correlation with EDSS. However, there was no significant correlation with the disease duration. Moreover, there was an inverse correlation between the SDMT and MRI variables involving the number of cortical lesions, different cortical lesion shapes, various subtypes of cortical lesions, and the assessed total lesion load (Table 3). SPMS patients had impaired cognition (mean $\pm \mathrm{SD}=6.3 \pm 1.3$ ) more severe than RRMS patients (mean $\pm \mathrm{SD}=8.7 \pm$ 2.7 ) with significant value $(p=0.003)$. On multivariate analysis, only the wedge-shaped cortical lesion is the independent predictor for MoCA $(p=0.006)$. However, both Curvilinear and oval-shaped lesions were independent predictors for SDMT ( $p=0.006,0.004$ respectively).

\section{Discussion}

Although there is large evidence from the literature that cortical pathology is correlated with disability and cognition, cognitive dysfunction is still not included in the routine clinical assessment of MS patients. Recently, MS treatment goals have become more ambitious aiming at "no evidence of disease activity" (NEDA). Optimally, NEDA must incorporate measures that reflect inflammatory and degenerative components of MS and factors that reflect disease impact including cognitive impairment. Thus, it is practically very important to monitor cortical involvement in MS patients during their routine follow-up assessment. In this study, it was found that the sensitivity of the MoCA test in the detection of cognitive deficit in MS patients (66.7\%) was higher than that of SDMT (43.3\%). This could be explained as the MoCA test that reflects cognitive functioning more broadly across multiple domains while SDMT is used to assess only one domain (information processing speed) which is not specifically measured by the MoCA. Besides, a previous large-scale study recommended that it was better to combine both MoCA and SDMT scales in the cognitive assessment of MS patients [10]. Interestingly, we found an excellent inter-observer correlation of number, subtypes, and both curvilinear and oval shapes of the cortical lesions and total lesion load indicating reliability and accuracy of the DIR sequence. Two comparative previous studies confirmed the superiority of DIR to conventional sequences (FLAIR and T2) in the detection
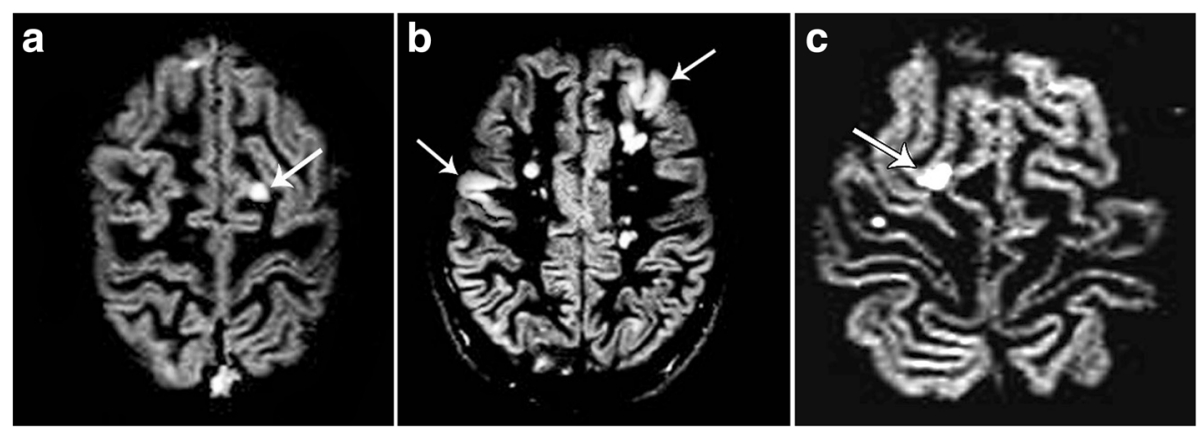

Fig. 2 Axial DIR images showing cortical lesion morphology: a Oval-shaped cortical lesion in the left frontal region. b Two curvilinear-shaped cortical lesions in the right frontoparietal and left frontal regions. c A wedge-shaped cortical lesion in the right frontal region 
Table 2 MRI (DIR sequence) findings of the studied group as noted by both observers

\begin{tabular}{lll}
\hline & $\begin{array}{l}\text { Observer 1 } \\
\boldsymbol{n} \text { of lesions (\%) }\end{array}$ & $\begin{array}{l}\text { Observer 2 } \\
\boldsymbol{n} \text { of lesions (\%) }\end{array}$ \\
\hline $\begin{array}{ll}\text { Cortical lesion subtype } \\
\text { IC }^{\mathbf{a}}\end{array}$ & $181(33.5)$ & $225(35.5)$ \\
$\mathrm{LC}^{\mathrm{b}}$ & $177(32.5)$ & $172(27.2)$ \\
$\mathrm{JC}^{\mathrm{c}}$ & $186(34.2)$ & $236(37.3)$ \\
Cortical lesion shape & & \\
Curvilinear & $57(10.5)$ & $77(12.2)$ \\
Oval & $264(48.5)$ & $313(49.4)$ \\
Wedge & $223(41)$ & $243(38.4)$ \\
\hline
\end{tabular}

antracortical

beukocortical

'Juxtacortical

of cortical and also white matter lesions in MS. DIR showed significantly higher lesion load in both supraand infratentorial locations $[23,24]$. The total number of cortical lesions assessed in this study was negatively correlated with scores of cognitive assessment tests. A recent similar study has reported a strong significant correlation between cortical lesion numbers detected by DIR and poor performance on cognitive assessment tests [25]. Moreover, a study of a total of 39 MS patients revealed that the severity of cognitive impairment was negatively correlated with EDSS and cortical lesion numbers [26].

The most common type of cortical lesions noted by both observers in the current research was JC followed by IC then LC. We found that LC lesions were significantly correlated with cognitive scores of either MoCA or SDMT. Besides, previous studies reported that LC lesions have a strong impact on cognition, maybe due to disruption of subcortical $U$ fibers and thus impaired regional cortico-subcortical connectivity [26, 27]. The most common shape of 633 cortical lesions observed in our study using DIR was oval $49.4 \%$ followed by wedge $38.4 \%$ then curvilinear $12.2 \%$. Although all cortical lesion shapes were significantly correlated with cognitive scores of either MoCA or SDMT, we found that oval-shaped lesions were slightly highly significant than other types. Moreover, on multivariate analysis, the only wedgeshaped cortical lesion was considered an independent predictor for MoCA while both curvilinear and ovalshaped lesions were considered as independent predictors for SDMT. However, one previous study reported that using DIR, 765 CGM lesions were initially classified as $86.9 \%$ oval, $7.7 \%$ curvilinear, and $5.3 \%$ wedge-shaped. While using phase-sensitive inversion recovery (PSIR), a high number of both oval and wedge-shaped lesions were reclassified into the curvilinear shape. Thus, curvilinear lesions were identified in $7.7 \%$ of MS patients using DIR, and $23.6 \%$ of them using PSIR in that study. So, they recommended that it was important to identify the shape of cortical lesions and suggested that curvilinear lesions were more specific for neuroinflammation while wedge-shaped lesions were related to vascularassociated factors [20]. In the current work, the total lesion load has been negatively correlated with MoCA and SDMT cognitive scores. Previous studies detected a negative correlation between SDMT and the total lesion load [28, 29]. Moreover, another study assessed the role of multiple MRI parameters, expressed as a fraction (f) of intracranial volume as WM-f, GM- $\mathrm{f}$, and abnormal WM (AWM-f), a measure of lesion load in predicting long-term cognitive impairment in a cohort of MS patients; they found that lesion load was the best predictor of cognitive impairment in MS patients. In particular, AWM-f was predictive of an impaired SDMT performance [30]. That was explained by another study that reported a significant correlation between cognitive impairment in MS and total lesion load using MRI 3.0 T. As, it improved detection of small lesions missed by the conventional MRI, particularly those in the periventricular white matter, cortical, or juxtacortical areas [31]. The latter study suggested that these lesions could result in cortical-subcortical circuit disconnection involved in complex cognitive functions. However, WM
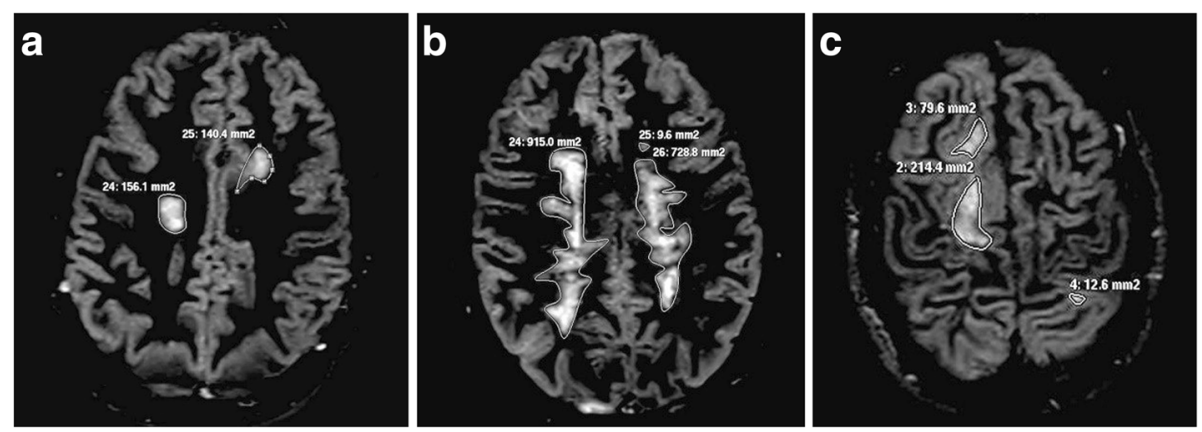

Fig. 3 Axial DIR images showing contouring and segmentation method of MS plaques for calculation of total lesion load 
Table 3 Correlation between MoCA and SDMT and other patients' variables

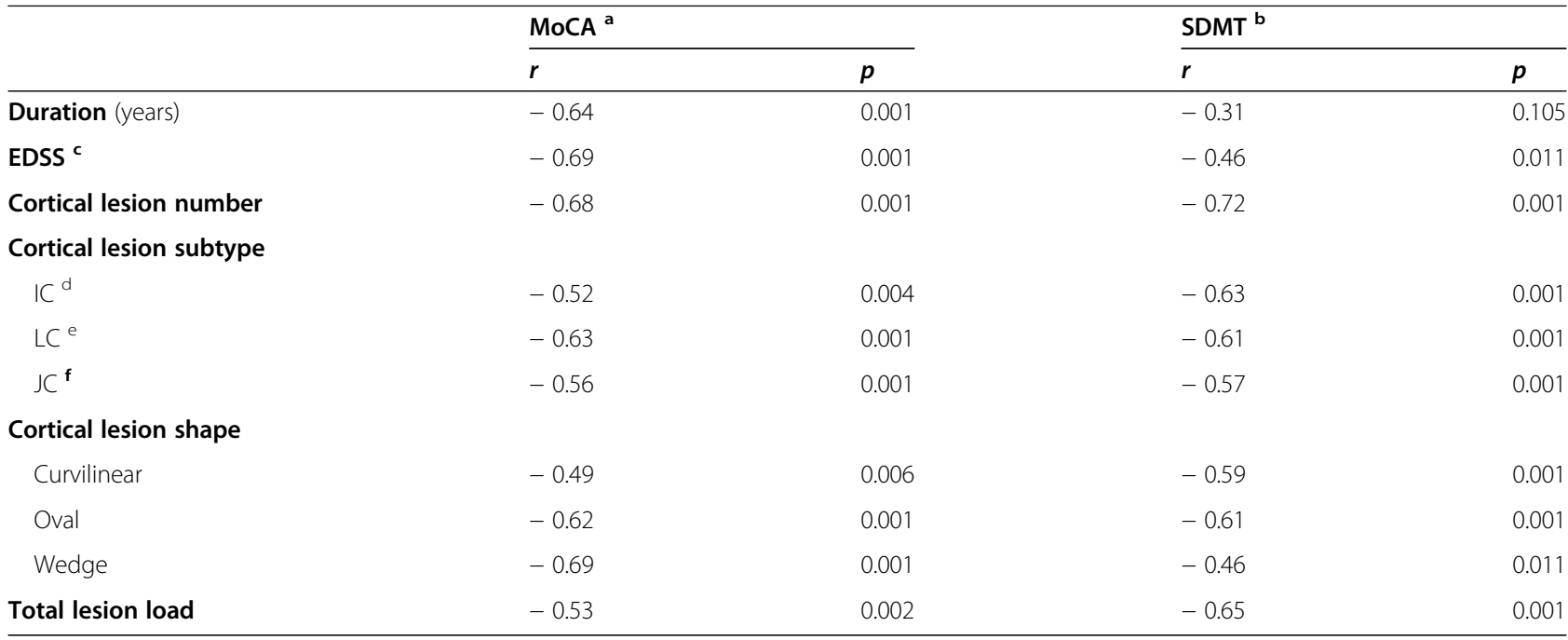

${ }^{a}$ Montreal cognitive assessment

bSymbol digital modality test

CExpanded disability status scale

${ }^{\mathrm{d}}$ Intracortical

ELeukocortical

fJuxtacortical

abnormalities alone cannot fully explain the extent of clinical symptoms and cognitive impairment in MS. The importance of white matter lesion location; gray matter lesions; and cortical and subcortical grey matter brain atrophy gives a stronger correlation with the severity of cognitive impairment [32]. In our study, we used regression analysis for variables interacting with MoCA as age, the age of onset, years of education, disease duration, EDSS, the pattern of cortical lesions, and total lesion load. We found that the only wedge-shaped cortical lesion was considered an independent predictor for
MoCA. However, a previous study used regression analysis to determine whether age, body mass index (BMI), EDSS, fatigue, and mood predicted MoCA scores. They found that only EDSS was uniquely predictive of MoCA scores [10].

However, there are a few limitations to this study. First, this study was carried upon a 1.5-Tesla scanner. Application of the DIR technique on a higher 3-Tesla scanner combined with other imaging sequences such as diffusion tensor imaging and phase-sensitive inversion recovery will improve the results. Second, this study

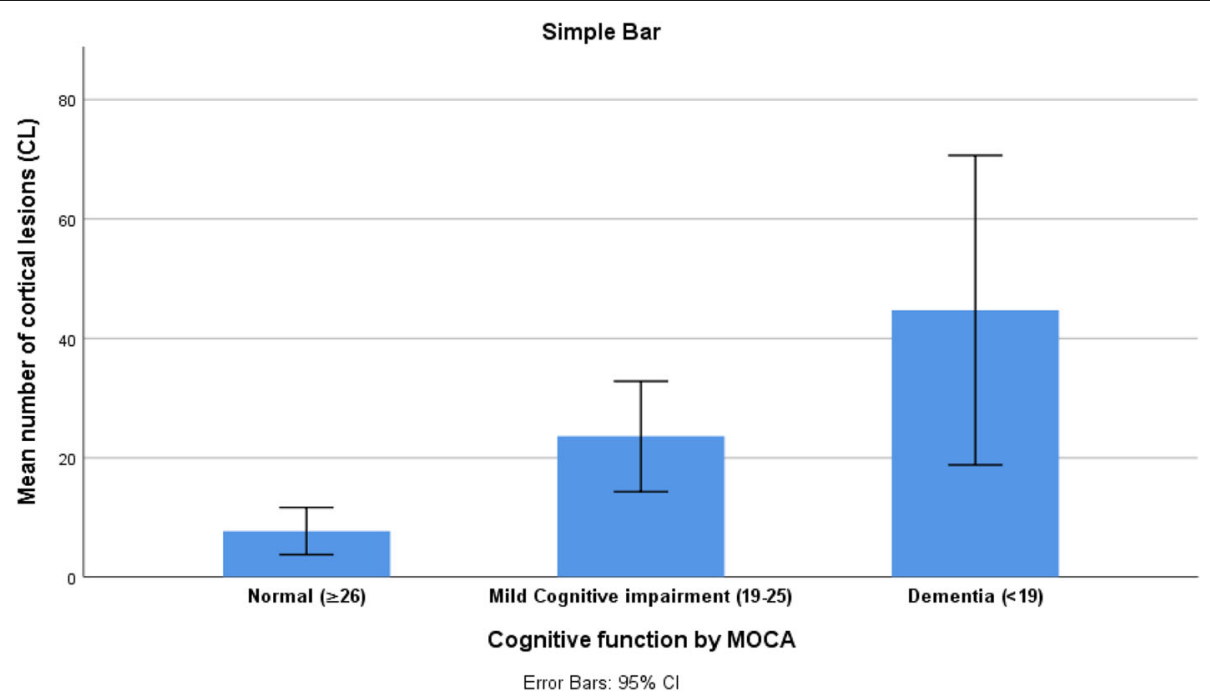

Fig. 4 Simple bar chart revealing mean $C L$ numbers in different cognitive status assessed by MoCA test 
includes a small number of patients. Further studies are recommended upon a large scale of patients for more valid results.

\section{Conclusion}

In conclusion, DIR can detect cortical lesions of MS which were well correlated with cognitive dysfunction as well as disability progression in these patients. Thus, DIR is found to be reliable and useful for clinical purposes to suspect cognitive dysfunction in MS patients. Therefore, we strongly recommend adding a DIR sequence in the routine MR protocols of multiple sclerosis, and we also recommend incorporating the assessment of cortical involvement and cognition into the essential items when making treatment decisions in MS patients especially in this era of the abundance of high efficacy disease-modifying therapies.

\section{Abbreviations}

MS: Multiple sclerosis; RRMS: Relapsing-remitting multiple sclerosis; SPMS: Secondary progressive multiple sclerosis; EDSS: Expanded Disability Status Scale; MoCA: Montreal Cognitive Assessment; SDMT: Symbol Digit Modality Test; MCl: Mild cognitive impairment; DIR: Double inversion recovery; IC: Intracortical; LC: Leukocortical; JC: Juxtacortical; PSIR: Phasesensitive inversion recovery; NEDA: No evidence of disease activity

\section{Acknowledgements}

Not applicable

\section{Authors' contributions}

SS collected the data and analyzed it including the statistical analysis. BT analyzed the data, drafted the manuscript for intellectual content, and was a major contributor in writing the manuscript. AA and EA analyzed the data and drafted the manuscript for intellectual content, and the authors read and approved the final manuscript.

\section{Funding}

This research did not receive any specific grant from funding agencies in the public, commercial, or not-for-profit sectors.

\section{Availability of data and materials}

The datasets generated during and/or analyzed during the current study are available from the corresponding author on reasonable request.

\section{Ethics approval and consent to participate}

This study was conducted following the guidelines of the 1995 Declaration of Helsinki and approved by the local institutional review board (IRB), approval number: R.MS/17.03.24. Written and verbal consent were obtained from all the study participants.

\section{Consent for publication}

All patients signed consents by themselves for publishing their cases.

\section{Competing interests}

The authors declare no competing interest.

\section{Author details}

${ }^{1}$ Department of Neurology, Mansoura Faculty of Medicine, Mansoura, Egypt. ${ }^{2}$ Department of Diagnostic Radiology, Mansoura Faculty of Medicine, Mansoura, Egypt.
Received: 19 November 2020 Accepted: 5 February 2021

Published online: 22 February 2021

\section{References}

1. Trenova AG, Slavov GS, Manova MG, Aksentieva JB, Miteva LD, Stanilova SA. Cognitive impairment in multiple sclerosis. Folia Med (Plovdiv). 2016;58(3): 157-63.

2. Papadopoulou A, Müller-Lenke N, Naegelin Y, Kalt G, Bendfeldt K, Kuster P, et al. Contribution of cortical and white matter lesions to cognitive impairment in multiple sclerosis. Mult Scler. 2013;19(10):1290-6.

3. Geurts Jل Jalabrese M, Fisher E, Rudick RA. Measurement and clinical effect of grey matter pathology in multiple sclerosis. Lancet Neurol. 2012;11(12): 1082-92.

4. Filippi M, Rocca MA, Calabrese M, Sormani MP, Rinaldi F, Perini P, et al. Intracortical lesions: relevance for new MRI diagnostic criteria for multiple sclerosis. Neurology. 2010;75(22):1988-94

5. Fischer MT, Wimmer I, Höftberger R, Gerlach S, Haider L, Zrzavy T, et al. Disease-specific molecular events in cortical multiple sclerosis lesions. Brain. 2013;136(6):1799-815.

6. Thompson AJ, Banwell BL, Barkhof F, Carroll WM, Coetzee T, Comi G, et al. Diagnosis of multiple sclerosis: 2017 revisions of the McDonald criteria. Lancet Neurol. 2018;17(2):162-73.

7. Rimkus CM, Schoonheim MM, Steenwijk MD, Vrenken H, Eijlers AJ, Killestein J, et al. Gray matter networks and cognitive impairment in multiple sclerosis. Mult Scler. 2019;25(3):382-91.

8. Calabrese M, Poretto V, Favaretto A, Alessio S, Bernardi V, Romualdi C, et al. Cortical lesion load associates with progression of disability in multiple sclerosis. Brain. 2012;135(10):2952-61.

9. Miller E, Morel A, Redlicka J, Miller I, Saluk J. Pharmacological and nonpharmacological therapies of cognitive impairment in multiple sclerosis. Curr Neuropharmacol. 2018;16(4):475-83.

10. Charvet LE, Taub E, Cersosimo B, Rosicki C, Melville P, Krupp LB. The Montreal cognitive assessment (MoCA) in multiple sclerosis: relation to clinical features. J Mult Scler. 2015;2(135):2376-0389.

11. Parmenter BA, Weinstock-Guttman B, Garg N, Munschauer F, Benedict RH Screening for cognitive impairment in multiple sclerosis using the Symbol Digit Modalities Test. Mult Scler. 2007;13(1):52-7.

12. Elkholy SF, Sabet MA, Mohammad ME, Asaad RE. Comparative study between double inversion recovery (DIR) and fluid-attenuated inversion recovery (FLAIR) MRI sequences for detection of cerebral lesions in multiple sclerosis. Egypt J Radiol Nuclear Med. 2020;51(1):1-0.

13. Favaretto A, Poggiali D, Lazzarotto A, Rolma G, Causin F, Gallo P. The paralle analysis of phase sensitive inversion recovery (PSIR) and double inversion recovery (DIR) images significantly improves the detection of cortical lesions in multiple sclerosis (MS) since clinical onset. Plos One. 2015;10(5):e0127805.

14. Simon B, Schmidt S, Lukas C, Gieseke J, Träber F, Knol DL, et al. Improved in vivo detection of cortical lesions in multiple sclerosis using double inversion recovery MR imaging at 3 Tesla. Eur Radiol. 2010;20(7):1675-83.

15. Kurtzke JF. Rating neurologic impairment in multiple sclerosis: an expanded disability status scale (EDSS). Neurology. 1983;33(11):1444-52.

16. Nasreddine ZS, Phillips NA, Bédirian V, Charbonneau S, Whitehead V, Collin I, et al. The Montreal Cognitive Assessment, MoCA: a brief screening tool for mild cognitive impairment. J Am Geriatr Soc. 2005;53(4):695-9.

17. Milani SA, Marsiske M, Cottler LB, Chen X, Striley CW. Optimal cutoffs for the Montreal Cognitive Assessment vary by race and ethnicity. Alzheimers Dement (Amst). 2018;10:773-81.

18. Smith A. The symbol-digit modalities test: a neuropsychologic test of learning and other cerebral disorders. Learn Disord. 1968;3:83-91.

19. Geurts JJ, Roosendaal SD, Calabrese M, Ciccarelli O, Agosta F, Chard DT, et al. Consensus recommendations for MS cortical lesion scoring using double inversion recovery MRI. Neurology. 2011;76(5):418-24.

20. Sethi V, Muhlert N, Ron M, Golay X, Wheeler-Kingshott CA, Miller DH, et al. MS cortical lesions on DIR: not quite what they seem? Plos One. 2013;8(11):e78879.

21. Filippi M, Rocca MA, Ciccarelli O, De Stefano N, Evangelou N, Kappos L, et al. MRI criteria for the diagnosis of multiple sclerosis: MAGNIMS consensus guidelines. Lancet Neurol. 2016;15(3):292-303.

22. Parodi RC, Sardanelli F, Renzetti P, Rosso E, Losacco C, Ferrari A, et al. Growing region segmentation software (GRES) for quantitative magnetic resonance imaging of multiple sclerosis: intra-and inter-observer agreement variability: a comparison with manual contouring method. Eur Radiol. 2002;12(4):866-71. 
23. Elnekeidy AM, Kamal MA, Elfatatry AM, Elskeikh ML. Added value of double inversion recovery magnetic resonance sequence in detection of cortical and white matter brain lesions in multiple sclerosis. Egypt J Radiol Nuclear Med. 2014;45(4):1193-9.

24. Abidi Z, Faeghi F, Mardanshahi Z, Mortazavi H. Assessment of the diagnostic accuracy of double inversion recovery sequence compared with FLAIR and T2W_TSE in detection of cerebral multiple sclerosis lesions. Electron Physician. 2017:9(4):4162-70.

25. Matsushita F, Kida H, Tabei Kl, Nakano C, Matsuura K, li Y, et al. Clinical significance of cortical lesions in patients with multiple sclerosis: a neuropsychological and neuroimaging study. Brain Behav. 2018;8(3):e00934

26. Nelson F, Datta S, Garcia N, Rozario NL, Perez F, Cutter G, et al. Intracortical lesions by $3 \mathrm{~T}$ magnetic resonance imaging and correlation with cognitive impairment in multiple sclerosis. Mult Scler. 2011;17(9):1122-9.

27. Harrison DM, Roy S, Oh J, Izbudak I, Pham D, Courtney S, et al. Association of cortical lesion burden on 7-T magnetic resonance imaging with cognition and disability in multiple sclerosis. JAMA Neurol. 2015;72(9):100412

28. Deloire MS, Ruet A, Hamel D, Bonnet M, Dousset V, Brochet B. MRI predictors of cognitive outcome in early multiple sclerosis. Neurology. 2011; 76(13):1161-7.

29. Rossi F, Giorgio A, Battaglini M, Stromillo ML, Portaccio E, Goretti B, et al. Relevance of brain lesion location to cognition in relapsing multiple sclerosis. Plos one. 2012;7(11):e44826.

30. Patti F, De Stefano M, Lavorgna L, Messina S, Chisari CG, Ippolito D, et al Lesion load may predict long-term cognitive dysfunction in multiple sclerosis patients. Plos One. 2015;10(3):e0120754.

31. Stankiewicz JM, Glanz BI, Healy BC, Arora A, Neema M, Benedict RH, et al. Brain MRI lesion load at 1.5 T and $3 \mathrm{~T}$ versus clinical status in multiple sclerosis. J Neuroimaging. 2011;21(2):e50-6.

32. Sumowski JF, Benedict R, Enzinger C, Filippi M, Geurts JJ, Hamalainen P, et al. Cognition in multiple sclerosis: state of the field and priorities for the future. Neurology. 2018;90(6):278-88.

\section{Publisher's Note}

Springer Nature remains neutral with regard to jurisdictional claims in published maps and institutional affiliations.

\section{Submit your manuscript to a SpringerOpen ${ }^{\circ}$ journal and benefit from:}

- Convenient online submission

- Rigorous peer review

- Open access: articles freely available online

- High visibility within the field

- Retaining the copyright to your article

Submit your next manuscript at $\boldsymbol{\nabla}$ springeropen.com 